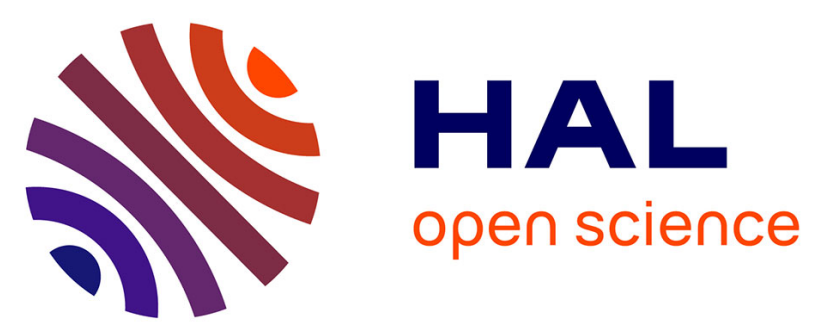

\title{
Prognostic value of the texture analysis parameters of the initial computed tomographic scan for response to neoadjuvant chemoradiation therapy in patients with locally advanced rectal cancer
}

Benjamin Vandendorpe, Carole Durot, Loïc Lebellec, Marie-Cécile Le Deley, Dienabou Sylla, André-Michel Bimbai, Kocéila Amroun, Fabrice Ramiandrisoa, Abel Cordoba, Xavier Mirabel, et al.

\section{- To cite this version:}

Benjamin Vandendorpe, Carole Durot, Loïc Lebellec, Marie-Cécile Le Deley, Dienabou Sylla, et al.. Prognostic value of the texture analysis parameters of the initial computed tomographic scan for response to neoadjuvant chemoradiation therapy in patients with locally advanced rectal cancer. Radiotherapy \& Oncology, 2019, 135, pp.153-160. 10.1016/j.radonc.2019.03.011 . hal-02483781

\author{
HAL Id: hal-02483781 \\ https://hal.science/hal-02483781
}

Submitted on 22 Oct 2021

HAL is a multi-disciplinary open access archive for the deposit and dissemination of scientific research documents, whether they are published or not. The documents may come from teaching and research institutions in France or abroad, or from public or private research centers.
L'archive ouverte pluridisciplinaire HAL, est destinée au dépôt et à la diffusion de documents scientifiques de niveau recherche, publiés ou non, émanant des établissements d'enseignement et de recherche français ou étrangers, des laboratoires publics ou privés.

\section{(ㅇ)(1) $\$$}

Distributed under a Creative Commons Attribution - NonCommerciall 4.0 International 
1 Prognostic value of the texture analysis parameters of the initial computed

2 tomographic scan for response to neoadjuvant chemoradiation therapy in

4 Benjamin Vandendorpe ${ }^{a 1}$, Carole Durot ${ }^{b 1}$, Loïc Lebellec ${ }^{c}$, Marie-Cécile Le Deley ${ }^{c, d}$,

5 Dienabou Sylla ${ }^{c}$, André-Michel Bimbaic, Kocéila Amroune, Fabrice Ramiandrisoa ${ }^{\mathrm{a}}$,

6 Abel Cordoba ${ }^{\dagger}$, Xavier Mirabel ${ }^{\dagger}$, Christine Hoeffel ${ }^{b}$, David Pasquier ${ }^{\dagger}$, Stéphanie

7 Servagi-Vernat ${ }^{a, 9}$

8

9

Conflicts of interest: none

${ }^{1}$ Equal contribution.

Corresponding author:

Dr Stéphanie Servagi Vernat 1 rue Koenig, CS80014

51726 REIMS

aDepartment of Radiation Oncology, Institut Jean Godinot, Reims France,

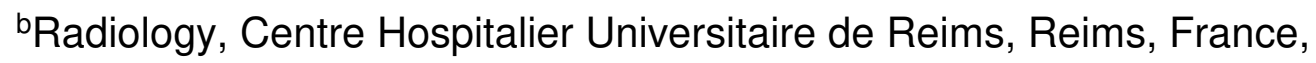

'Biostatistics unit, Centre Oscar Lambret, Lille, France, dCESP, INSERM, Paris-Sud Paris-Orsay University, Villejuif, France, eDepartment of surgery, Institut Jean Godinot, Reims France

fDepartment of Radiation Oncology, Centre Oscar Lambret, Lille, France, gCRESTIC, University of Reims, Reims, France.

Department of Radiation Oncology, Institut Jean Godinot,

Tel: +3326504177, Fax +3326504369

Email: stephanie.servagi@gmail.com

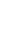


1 Abbreviations: TA: texture analysis; SSF: spatial scale filtration ; Mpp: mean positive

2 pixel ; CEA: carcinoembryonic antigen; Cl: confidence Interval

3

4

5

6 
1 Abstract:

2 Background and Purpose

3 Baseline contrast-enhanced computed tomography (CT)-derived texture analysis in

4 locally advanced rectal cancer could help offer the best personalized treatment. The

5 purpose of this study was to determine the value of baseline-CT texture analysis in

6 the prediction of downstaging in patients with locally advanced rectal cancer.

7 Patients and methods

8 We retrospectively included all consecutive patients treated with neoadjuvant

9 chemoradiation therapy (CRT) followed by surgery for locally advanced rectal cancer.

10 Tumor texture analysis was performed on the baseline pre-CRT contrast-enhanced

11 CT examination. Based on the selected model of downstaging with a penalized 12 logistic regression in a training set, a radiomics score (Radscore) was calculated as a 13 linear combination of selected features. A multivariable prognostic model that 14 included Radscore and clinical factors was created.

15 Results

16 Of the 121 patients included in the study, 109 patients $(90 \%)$ had T3-T4 cancer and 1799 (82\%) had $\mathrm{N}+$ cancer. A downstaging response was observed in 96 patients 18 (79\%). In the training set (79 patients), the best model (ELASTIC-NET method) 19 reduced the 36 texture features to a combination of 6 features. The multivariate 20 analysis retained the Radscore (odds ratio $[O R]=13.25 ; 95 \%$ confidence interval $21[95 \% \mathrm{Cl}], 4.06-71.64 ; \mathrm{p}<0.001)$ and age $(\mathrm{OR}=1.10 / 1$ year; $1.03-1.20 ; \mathrm{p}=0.008)$ as 
1 independent factors. In the test set, the area under the curve was estimated to be

$2 \quad 0.70(95 \% \mathrm{Cl}, 0.48-0.92)$.

3 Conclusion

4 This study presents a prognostic score for downstaging, from initial computed

5 tomography-derived texture analysis in locally advanced rectal cancer, which may

6 lead to a more personalized treatment for each patient.

7

8 Keywords: Computed Tomography, texture analysis, prognostic value, neoadjuvant

9 therapy, rectal cancer 


\section{Introduction}

3 The standard treatment for locally advanced rectal cancer is neoadjuvant

4 chemoradiation therapy (CRT) followed by total mesorectal excision (TME) with a 6-

5 10-week interval between these two treatments [1] [2]. Patients with a complete pathological response $(\mathrm{pCR})$ after CRT have better long-term outcomes than those

7 without a pCR. pCR might be indicative of a favorable biological tumor profile with a lower propensity for local or distant recurrence and an improved survival [3]. The tumor response to CRT may influence the prognosis after surgery. Beddy et al reported that the 5-year disease-free survival after CRT was significantly better in tumor regression patients than in those with no tumor regression [4]. Computed tomography (CT) is commonly used for tumor staging and dosimetric radiotherapy study. It may provide prognostic information based on texture analysis (TA) [5]. TA is an emerging method that can reflect the degree of tumor heterogeneity. It is indeed difficult to visually assess tumor heterogeneity on a CT scan, because of photon noise and inter-observer variability in the interpretation of imaging. CT texture analysis software can address these drawbacks and quantify heterogeneity in a defined area objectively [6] [7]. CT texture analysis is an important research focus in many types of cancer [8][9][10]. A recent study predicted complete response after neo-adjuvant chemoradiation for locally advanced rectal cancer using radiomics from initial CT using a deep neural network approach involving 1683 radiological features [11]. Textural analysis of baseline ${ }^{18} \mathrm{FDG}$ PET/CT provides strong independent prognostic factors of survival in patients with locally advanced rectal cancer [12]. MRI-based texture analysis of rectal cancers may be associated with poor response to pre-operative treatment and could potentially help in patient selection for 
1 individualized therapy [13]. Contrast-enhanced CT of the thorax, abdomen, and

2 pelvis is always performed on patients with rectal cancer before the start of CRT and

3 during follow-up, whereas MRI is only indicated for patients with low and middle

4 rectal tumors.

5 The present study aimed to develop and validate a prognostic model that includes a

6 radiomics score based on baseline contrast-enhanced CT texture analysis,

7 performed by a single expert radiologist, for downstaging in patients with locally

8 advanced rectal cancer.

\section{$9 \quad$ Patients and methods}

\section{Patients}

11 This retrospective bicentric study complies with the "reference methodology" MR003 adopted by the French Data Protection Authority (CNIL) and patients did not object to the use of their clinical data for the research purpose.

All consecutive patients with locally advanced rectal cancer who received CRT based on fluorouracil followed by surgery between 2011 and 2017 at the Oscar Lambret Centre or Jean Godinot Institute were eligible for this study. Patients were identified from the hospital databases. CRT involved delivery of 45-50 Gy in 5 fractions per week combined with administration of capecitabine only, both capecitabine and metformine, or both leucovorine and 5-fluorouracil (LV-5FU 2 ). Patients with a metastatic disease at baseline, who underwent a different chemotherapy regimen, or who were finally not operated on were not eligible for participation in this study. Additionally, patients for whom texture analysis of the baseline CT scan was not feasible or pathological evaluation of the surgical specimen was not performed, and patients who refused to participate in the study were excluded. Clinical data were retrospectively extracted from the hospital medical records. 
2 The study endpoint of the prognostic factor analysis was downstaging (yes versus

3 no), which is defined as a decrease in T-staging and or $\mathrm{N}$-staging without an increase

4 of the second component, when comparing the pathological staging (ypTNM) to the

5 initial staging (TNM).

6 Candidate variables evaluated in the prognostic models were age, initial tumor size,

7 initial tumor and nodal staging (TNM $7^{\text {th }}$ edition), initial staging, time interval between CRT and surgery ( $\leq 7$ versus $>7$ weeks), tumor site, World Health Organization Performance status (WHO: 0 versus 1), and radiomic features.

$\underline{\text { Texture analysis }}$

Tumor texture analysis was performed on contrast-enhanced CT images acquired at a portal phase (diagnostic or dosimetric CT), using TexRAD ${ }^{\circledR}$ software $\left(T_{e x R A D}{ }^{\circledR}\right.$ Ltd), by a single expert radiologist. A radiologist specialized in gastro-intestinal imaging chose the CT slice where the tumor was best visualized. The chosen slices were then exported in DICOM format and anonymized. The region of interest was manually delineated around the tumor. CT texture analysis was performed in a twostep process including image filtration and statistical quantification. The principle behind texture analysis is detailed in Supplementary data-1. Five spatial scale image filtrations (SSF) features were selectively extracted corresponding to different anatomic scales: fine (SSF2, object radius of $2 \mathrm{~mm}$ ), medium (SSF3-5, object radius of 3-5 $\mathrm{mm}$ ), and coarse (SSF6, object radius of $6 \mathrm{~mm}$ ) texture scales, by using a Laplacian of Gaussian special band pass filter (Figure 1). In addition, the unfiltered images (SSF0) corresponded to conventional CT images. For each filtration, six measures were used to summarize the distribution of pixels and tumor heterogeneity: mean grey-level intensity (mean), standard deviation (sd), entropy (irregularity of pixel intensities in space), mean positive pixel (mpp), skewness (symmetry of the 
1 histogram distribution), and kurtosis (measure of whether the data are heavy-tailed or

2 light-tailed relative to a normal distribution) [14].

$3 \quad$ Statistical analysis

4 The association between clinicopathologic data and downstaging was assessed, 5 using the chi-square or Fisher exact test for categorical variables and the Student t6 test for continuous variables. Radiomics features were also compared between the 7 two groups. Additionally, correlation between the 36 radiomic features was evaluated 8 (correlation matrix).

9 The prognostic model was developed on a training sample, and then tested on a "test" sample. Although this study was bicentric and involved two independent

11 cohorts, a random sampling of the population was needed to obtain a training set 12 representing $2 / 3$ of the overall study population, as only 19 patients were recruited in 13 the second center.

14 Considering the high-dimensional data and correlation between these data, selection of radiomic features associated with downstaging was first performed using penalized logistic regression in the training set [15]. We searched the best penalized

17 regression between the Ridge $(\alpha=0)$, ELASTIC-NET (with $\alpha$ varying from 0.1 to 0.9 ), and least absolute, shrinkage, and selection operator (LASSO; $\alpha=1$ ) methods. A seven-fold cross validation provided the $\alpha$-value and minimum value of the tuning parameter $\lambda$ of these methods and maximized the area under the curve (AUC) of the

21 receiver operating characteristic curve $(\mathrm{ROC})$. Based on the selected model, a 22 radiomics score (Radscore) was calculated as a linear combination of selected 23 features weighted by their regression coefficients. 
1 The association of the radiomics score (Radscore) with downstaging was assessed

2 in the training sample and in the overall cohort by using the Student t-test. The ROC

3 curve was plotted to estimate the AUC in the training data set.

4 Univariable logistic regression analysis was performed to select variables for the first

5 step of the multivariable model $(p<0.20)$. Fractional polynomial models were used to

6 evaluate log-linearity of continuous variables. A step-wise selection provided the best

7 regression model based on Akaike's information criterion. All odds ratios (OR) were estimated with their $95 \%$ confidence interval $(95 \% \mathrm{Cl})$.

Performance of the final model built on the training set was then evaluated on the test data set through the ROC curve and the corresponding AUC with its $95 \% \mathrm{Cl}$.

The calibration of the final model was estimated with the Hosmer-Lemeshow test (a significant test statistic implies that the model does not calibrate perfectly) [16].

Statistical analysis was conducted with Stata software (version 15.0) and R software

(version 3.5.1). The packages used in $\mathrm{R}$ in this study are reported in Supplementary

data-2. Unless otherwise mentioned, the reported statistical significance levels were all two-sided, with statistical significance set at 0.05 .

\section{Results}

\section{Patient characteristics}

Of the 179 patients who were eligible for participation in the study, 121 were finally included: 102 from the Oscar Lambret Centre and 19 from the Jean Godinot Institute (Supplementary data-3). Patient and tumor characteristics are presented in Table 1. The median age of the cohort was 62 (range, 28-82) years, and there were more 23 men $(n=70,58 \%)$. The tumor classification according to the tumor site was as 24 follows: $58(48 \%)$ tumors in the low rectum, $55(45 \%)$ in the middle rectum, and 8 (7\%) in the upper rectum. At diagnosis, 109 patients (90\%) had T3 or T4 cancer and 
$199(82 \%)$ had a lymph node involvement $\left(N_{+}\right)$according to the TNM $7^{\text {th }}$ edition. A

2 total of 50 Gy was delivered in 114 patients (94\%), with concomitant administration of

3 capecitabine or LV5FU2 in 115 patients (95\%). The median time interval between

4 CRT and surgery was $8(2.0-19.0)$ weeks. Ninety-nine patients (82\%) underwent an

5 anterior resection with total mesorectal excision (TME). The resection was deemed

6 complete for 114 patients (94\%). A downstaging was obtained in 96 patients (79\%),

7 including 24 patients with a complete response (ypTON0, 20\%).

8 The population set was split between the training set that included 79 patients (62

9 with downstaging) and the test set that included 42 patients (34 with downstaging).

10 Description of the two samples is available in Supplementary data-4.

11 Radiomic features

12 The radiomic features are summarized in a correlation matrix (Supplementary data-5).

13 Overall, there were few correlations between extracted features from unfiltered 14 images (SSF0) and extracted features from filtered images (SSF 2-6). Conversely, 15 for the filtered images (SSF 2-6), each measured parameter (mean, sd, entropy, 16 mpp, skewness, kurtosis) was highly correlated between the different filtrations. 17 Some features were associated with the downstaging status (Supplementary data-6).

18 Radiomics feature selection and radiomics score (Radscore) building

Based on the training set (79 patients), the best model (ELASTIC-NET with $\alpha=0.9$ ) reduced the 36 texture features to a combination of 6 potential prognostic factors

21 (Figure 2A, 2B): mean0, mpp0, mean2, entropy4, kurtosis5 and mean6. The formula 22 of the resulting Radscore is defined in Supplementary data-7.

23 Validation of radiomics score (Radscore) in the training data set

24 As expected, there was a significant difference in Radscore between patients with a 25 downstaging and those without a downstaging, in the entire cohort $(p<0.001)$ (Table- 
11 and Supplementary data-8) and in the training data set $(p<0.001)$. The AUC in the

2 training sample was good: $0.86(95 \% \mathrm{Cl}: 0.73-0.98)$.

3 Development of a prognostic model for downstaging status in the training data set

4 In univariable analysis, the potential factors associated with the downstaging status

5 were Radscore, age, initial tumor size, and initial nodal staging. The multivariate

6 logistic regression retained the Radscore $(\mathrm{OR}=13.25 ; 95 \% \mathrm{Cl}, 4.06-71.64 ; \mathrm{p}<0.001)$

7 and age $(\mathrm{OR}=1.10 / 1$ year; $95 \% \mathrm{Cl}, 1.03-1.20 ; \mathrm{p}=0.008)$ (Table-3) as independent

8 prognostic factors, whereas initial tumor size and initial nodal staging were no longer

9 associated with downstaging ( $p$-value $=0.99$ and 0.98 , respectively).

Performance of the final prognostic model in the training and test data sets

11 As expected, a good performance was observed in the training data set, with an AUC of $0.90(95 \% \mathrm{Cl}: 0.83-0.97)$. In the test data set, the AUC was estimated to be 0.70 (95\% Cl: 0.48-0.92) (Figure-3). The Hosmer-Lemeshow test yielded a nonsignificant statistic $(p=0.89)$, which suggested that there was no departure from perfect fit. Regarding the calibration in the test sample, we found a nonsignificant statistic $(p=1.0)$, likely related to a lack of power.

\section{Discussion}

We developed a prognostic model for downstaging including a radiomics score based on baseline contrast-enhanced CT texture analysis performed by a single expert radiologist in patients with locally advanced rectal cancer. We validated the radiomics score on a test population, which is recommended in good practices but is rather uncommon in the literature [17]. The advantage of CT is that it is commonly used for tumor staging and dosimetric radiotherapy study.

Tumors are heterogeneous on gross and cellular levels, as well as on genetic and phenotypic levels, with spatial heterogeneity in cellular density, angiogenesis, and 
1 necrosis. This heterogeneity may affect prognosis and treatment, as more

2 heterogeneous tumors may be associated with more biologically aggressive behavior and increased resistance to treatment. This heterogeneity cannot be taken into account by profiles based on the analysis of tumors performed on a sample of the tumor volume. Furthermore, the most abundant cell type might not necessarily predict the properties of mixed populations [5]. Texture analysis provides an objective and quantitative assessment of tumor heterogeneity by analyzing the distribution and relationship of pixel or voxel grey levels in the image [18]. It is performed on a sample of the tumor, but this sample is chosen to be as representative as possible of the tumor heterogeneity. CT texture analysis was performed in the largest crosssectional area of the lesions instead of in the whole tumor. Whole-tumor analysis may allow a more precise evaluation of tumor heterogeneity and may also improve reproducibility. However, underestimation of entropy values by using the largest cross-sectional area seems to be less than $7 \%$ when compared whole-tumor analysis [19]. Moreover, single slice CT-based texture analysis is a more simple tool to be used in daily routine practice. Texrad software has already proven its value in other tumor settings such as melanoma, or hepatocellular carcinoma [20][21].

Studies using MRI [22] and PET/CT [12] with logistic regression or a simpler artificial neural network to predict treatment response have been conducted, and these studies have reported a good accuracy $(A \cup C=0.9756$, for the $M R I$ model). Using a non-penalized Cox modelling, Jalil et al. observed that a lower mpp at fine texture from pre-treatment MRI texture analysis was an independent predictor for overall survival, disease-free survival, and relapse-free survival [13]. Bibault et al. recently conducted a study using deep learning and radiomics to predict complete response after neo-adjuvant chemoradiation for locally advanced rectal cancer using 1683 
1 features and reported an AUC of $0.72(0.65-0.87)$. The primary endpoint they

2 considered, i.e. complete response, can be useful to promote organ preservation, but

3 is not useful to propose an intensification of the therapeutic regimen, because it does not differentiate responders from non-responders. In addition, they used a different statistical approach to model the texture features, which were based on 1683 parameters. Notably, the sample size is slightly larger in our study (121 versus 95) and we split the study population to validate the model in a test set. Finally, the region of interest was not delineated by a radiologist specialized in rectal cancer [11].

After a TME, $20 \%$ of patients have sexual complications, $44 \%$ consider the functional result after surgery as bad because of stool fractionation, urge incontinence, or real fecal incontinence [23], which is why a prospective American study suggests that the quality of life (QoL) of patients may be better after an abdominoperineal resection that after a TME [24]. Constant impotence and definitive colostomy occur as functional consequences of TME and of the pelvic radiation. The anterior resection syndrome is now considered as a real handicap, with an associated score [25]. For the abovementioned reasons, many studies are attempting to identify a solution to avoid a systematic surgery.

Some studies have tested a strategy of therapeutic intensification for select patients. GRECCAR-12 trial and PRODIGE-23 trial compare neoadjuvant CT with folfirinox followed by preoperative CRT versus preoperative CRT alone in patients with locally advanced rectal cancer in terms of 3-year disease-free survival.

Our final model, which included Radscore and age, provides interesting information that could be used to develop a personalized therapeutic strategy. CT-based texture analysis could allow upstream selection of the poor responders who may benefit from 
1 intensification of neoadjuvant treatment. Therefore, our model, after a validation of

2 our model, which includes the Radscore, in a larger study, we could use it to predict

3 downstaging, and exercise a wait-and-see approach if a cCR is confirmed after CRT.

4 Conversely, if no downstaging is predicted, we could propose neo-adjuvant

5 chemotherapy or higher radiation dose after validation of this approach with the

6 GRECCAR12 (NTC02514278) and PRODIGE23 (NCT01804790) studies.

7

8 Our study has some limitations. It was a retrospective study with a relatively small

9 size. Following the recommendations, the study population was split into a training 10 set and a test set, representing $2 / 3$ and $1 / 3$ of the whole sample. Even if the 11 estimated $A \cup C$ in the test set $(A \cup C=0.70)$ is deemed satisfactory, the confidence 12 interval is wide. This should be considered as an exploratory study and validation on 13 a larger and independent cohort is needed. In addition, as all patients were treated 14 with chemoradiation therapy, we cannot disentangle the prognostic value from the predictive value of the model. Inter-observer reproducibility was not taken into account, as the CT slice was chosen by only one expert radiologist. CT texture analysis was performed in the largest cross-sectional area of the tumor and not on the whole tumor, which is probably more representative of the tumor heterogeneity. We did not include the infiltration of the mesorectal fascia and the tumor regression grade (TRG) into the variables in the model. Indeed, regarding infiltration of the 21 mesorectal fascia, some patients had undergone their imaging examinations (CT22 scan and MRI) outside of the University Hospital and the imaging results would have 23 been missing for too many patients. Moreover, we know that CT is limited for the 24 assessment of circumferential margin, particularly in case of low rectal tumors. TRG 25 is predictive of therapeutic response in rectal cancer patients after 
1 chemoradiotherapy. However, TRG classification is not routinely used. Furthermore,

2 various TRG systems have been suggested, and the categorization is prone to

3 relevant interobserver variability [26]. For example Kim et al compared the prognostic

4 validity of four different TRG systems in order to identify the ideal TRG system [27].

5 The modified Dworak TRG system for evaluation of entire tumors including regional

6 lymph nodes is a better predictor of survival than current TRG systems for evaluation

7 of the primary tumor alone [28].

8 Finally, pre-treatment carcinoembryonic antigen (CEA) level $>5 \mathrm{ng} / \mathrm{mL}$ is significantly 9 associated with a lower pathological complete response rate [29]. However, this association was not considered in our analysis because the CEA level was not 11 available for all patients.

In conclusion, our study presents a prognostic model for downstaging status after CRT, which includes a radiomics score based on pre-treatment contrast-enhanced

CT, in patients with locally advanced rectal cancer. This may be a useful tool to select patients with an initially poorer downstaging probability and to propose to them an intensification of treatment via neo-adjuvant chemotherapy or higher radiation dose. After prospective evaluation of this approach in a randomized clinical study, this method could be implemented to better personalize treatment. 


\section{Acknowledgements}

2 The authors thank Mohammed Sedki, from INSERM Villejuif, for assisting us with the

3 statistical analysis.

4 


\section{References}

[1] Gérard J-P, André T, Bibeau F, Conroy T, Legoux J-L, Portier G, et al. Rectal cancer: French Intergroup clinical practice guidelines for diagnosis, treatments and follow-up (SNFGE, FFCD, GERCOR, UNICANCER, SFCD, SFED, SFRO). Dig Liver Dis Off J Ital Soc Gastroenterol Ital Assoc Study Liver 2017;49:359-67. doi:10.1016/j.dld.2017.01.152. [2] Sebag-Montefiore D, Bujko K, Valentini V. Rectal cancer multidisciplinary management: evidences and future landscape. Radiother Oncol J Eur Soc Ther Radiol Oncol 2009;92:145-7. doi:10.1016/j.radonc.2009.06.026.

[3] Maas M, Nelemans PJ, Valentini V, Das P, Rödel C, Kuo L-J, et al. Long-term outcome in patients with a pathological complete response after chemoradiation for rectal cancer: $\mathrm{a}$ pooled analysis of individual patient data. Lancet Oncol 2010;11:835-44. doi:10.1016/S14702045(10)70172-8.

[4] Beddy D, Hyland JMP, Winter DC, Lim C, White A, Moriarty M, et al. A simplified tumor regression grade correlates with survival in locally advanced rectal carcinoma treated with neoadjuvant chemoradiotherapy. Ann Surg Oncol 2008;15:3471-7. doi:10.1245/s10434-008-0149-y.

[5] Marusyk A, Almendro V, Polyak K. Intra-tumour heterogeneity: a looking glass for cancer? Nat Rev Cancer 2012;12:323-34. doi:10.1038/nrc3261.

[6] Davnall F, Yip CSP, Ljungqvist G, Selmi M, Ng F, Sanghera B, et al. Assessment of tumor heterogeneity: an emerging imaging tool for clinical practice? Insights Imaging 2012;3:573-89. doi:10.1007/s13244-012-0196-6.

[7] Ganeshan B, Miles KA. Quantifying tumour heterogeneity with CT. Cancer Imaging Off Publ Int Cancer Imaging Soc 2013;13:140-9. doi:10.1102/1470-7330.2013.0015.

[8] Ciaravino V, Cardobi N, DE Robertis R, Capelli P, Melisi D, Simionato F, et al. CT Texture Analysis of Ductal Adenocarcinoma Downstaged After Chemotherapy. Anticancer Res 2018;38:4889-95. doi:10.21873/anticanres.12803.

[9] Fan T-W, Malhi H, Varghese B, Cen S, Hwang D, Aron M, et al. Computed tomography-based texture analysis of bladder cancer: differentiating urothelial carcinoma from micropapillary carcinoma. Abdom Radiol N Y 2018. doi:10.1007/s00261-018-1694-x. [10] Chaddad A, Daniel P, Desrosiers C, Toews M, Abdulkarim B. Novel Radiomic Features based on Joint Intensity Matrices for Predicting Glioblastoma Patient Survival Time. IEEE J Biomed Health Inform 2018. doi:10.1109/JBHI.2018.2825027.

[11] Bibault J-E, Giraud P, Durdux C, Taieb J, Berger A, Coriat R, et al. Deep Learning and Radiomics predict complete response after neo-adjuvant chemoradiation for locally advanced rectal cancer. Sci Rep 2018;8:12611. doi:10.1038/s41598-018-30657-6.

[12] Lovinfosse P, Polus M, Van Daele D, Martinive P, Daenen F, Hatt M, et al. FDG PET/CT radiomics for predicting the outcome of locally advanced rectal cancer. Eur J Nucl Med Mol Imaging 2018;45:365-75. doi:10.1007/s00259-017-3855-5. [13] Jalil O, Afaq A, Ganeshan B, Patel UB, Boone D, Endozo R, et al. Magnetic resonance based texture parameters as potential imaging biomarkers for predicting long-term survival in locally advanced rectal cancer treated by chemoradiotherapy. Colorectal Dis Off J Assoc Coloproctology G B Irel 2017;19:349-62. doi:10.1111/codi.13496.

[14] Miles KA, Ganeshan B, Hayball MP. CT texture analysis using the filtration-histogram method: what do the measurements mean? Cancer Imaging Off Publ Int Cancer Imaging Soc 2013;13:400-6. doi:10.1102/1470-7330.2013.9045. 
[15] Sauerbrei W, Royston P, Binder H. Selection of important variables and

2 determination of functional form for continuous predictors in multivariable model building.

3 Stat Med 2007;26:5512-28. doi:10.1002/sim.3148.

4 [16] Kramer AA, Zimmerman JE. Assessing the calibration of mortality benchmarks in critical care: The Hosmer-Lemeshow test revisited. Crit Care Med 2007;35:2052-6. doi:10.1097/01.CCM.0000275267.64078.BO. [17] Limkin EJ, Sun R, Dercle L, Zacharaki El, Robert C, Reuzé S, et al. Promises and challenges for the implementation of computational medical imaging (radiomics) in oncology. Ann Oncol Off J Eur Soc Med Oncol 2017;28:1191-206. doi:10.1093/annonc/mdx034.

[18] Lubner MG, Smith AD, Sandrasegaran K, Sahani DV, Pickhardt PJ. CT Texture Analysis: Definitions, Applications, Biologic Correlates, and Challenges. Radiogr Rev Publ Radiol Soc N Am Inc 2017;37:1483-503. doi:10.1148/rg.2017170056.

[19] Ng F, Kozarski R, Ganeshan B, Goh V. Assessment of tumor heterogeneity by CT texture analysis: can the largest cross-sectional area be used as an alternative to whole tumor analysis? Eur J Radiol 2013;82:342-8. doi:10.1016/j.ejrad.2012.10.023.

[20] Durot C, Mulé S, Soyer P, Marchal A, Grange F, Hoeffel C. Metastatic melanoma: pretreatment contrast-enhanced CT texture parameters as predictive biomarkers of survival in patients treated with pembrolizumab. Eur Radiol 2019. doi:10.1007/s00330-018-5933-x. [21] Brenet Defour L, Mulé S, Tenenhaus A, Piardi T, Sommacale D, Hoeffel C, et al. Hepatocellular carcinoma: CT texture analysis as a predictor of survival after surgical resection. Eur Radiol 2019;29:1231-9. doi:10.1007/s00330-018-5679-5.

[22] Liu Z, Zhang X-Y, Shi Y-J, Wang L, Zhu H-T, Tang Z, et al. Radiomics Analysis for Evaluation of Pathological Complete Response to Neoadjuvant Chemoradiotherapy in Locally Advanced Rectal Cancer. Clin Cancer Res Off J Am Assoc Cancer Res 2017;23:7253-62. doi:10.1158/1078-0432.CCR-17-1038.

[23] Panis Y. [Surgical treatment of rectal cancer: is the price to be paid too high?]. Gastroenterol Clin Biol 2004;28:153-4.

[24] Grumann MM, Noack EM, Hoffmann IA, Schlag PM. Comparison of quality of life in patients undergoing abdominoperineal extirpation or anterior resection for rectal cancer. Ann Surg 2001;233:149-56.

[25] Emmertsen KJ, Laurberg S. Low anterior resection syndrome score: development and validation of a symptom-based scoring system for bowel dysfunction after low anterior resection for rectal cancer. Ann Surg 2012;255:922-8. doi:10.1097/SLA.0b013e31824f1c21. [26] Chetty R, Gill P, Govender D, Bateman A, Chang HJ, Deshpande V, et al. International study group on rectal cancer regression grading: interobserver variability with commonly used regression grading systems. Hum Pathol 2012;43:1917-23. doi:10.1016/j.humpath.2012.01.020.

[27] Kim SH, Chang HJ, Kim DY, Park JW, Baek JY, Kim SY, et al. What Is the Ideal Tumor Regression Grading System in Rectal Cancer Patients after Preoperative Chemoradiotherapy? Cancer Res Treat Off J Korean Cancer Assoc 2016;48:998-1009. doi:10.4143/crt.2015.254.

[28] Dworak O, Keilholz L, Hoffmann A. Pathological features of rectal cancer after preoperative radiochemotherapy. Int J Colorectal Dis 1997;12:19-23.

[29] Tural D, Selcukbiricik F, Özturk MA, Yildiz O, Turna H, Erdamar S, et al. The relation between pathological complete response and clinical outcome in patients with rectal cancer. Hepatogastroenterology 2013;60:1365-70. 


\section{Figure legends}

Figure 1: Texture analysis method

4

A- Delineation of the region of interest

B- Texture analysis image at fine texture scale (SSF2)

C- Texture analysis image at medium texture scale (SSF4)

D- Texture analysis image at coarse texture scale (SSF6)

Abbreviations: SSF, spatial scale filtration

Figure 2: Texture features selection using the best penalized binary logistic regression method.

(A) Tuning parameter lambda $(\lambda)$ selection in the ELASTIC-NET model using 7-fold cross validation via minimum criteria. The AUC of the ROC curve was plotted against $\log (\lambda)$. The first dotted vertical line corresponds to the minimum lambda parameter maximizing the AUC ( $\lambda \mathrm{min})$. The selected model was built with a $(\lambda \min +1 \mathrm{se})$ value of 0.0392 , which is equivalent to a log $(\lambda)$ value of -3.239 (second dotted vertical line).

(B) ELASTIC-NET coefficient profiles of the 36 texture features. A coefficient profile plot was generated against the $\log (\lambda)$ sequence. A dotted line was drawn at the value selected in cross-validation $(\lambda \min +1 \mathrm{se})$, resulting in 6 nonzero coefficients.

These plots correspond to the ELASTIC-NET model with $\alpha=0.9$. 
2 Figure 3: Receiver operating characteristic (ROC) curves of the final model in 3 the training and in the test sets

4 Legend: The final model is logit $(p)=-7.48+2.58 \times$ Radscore $+0.10 \times$ Age (in year)

5 The plain curves correspond to the observed results. The dotted curves correspond 6 to the smoothed curves, obtained by bootstrap replicates. Area under the curve 7 (AUC) values are computed with the observed results. The grey line corresponds to 8 the performance that would be obtained at random.

9 
1 Tables

3 Table 1: Patient and treatment characteristics, overall and according to the response

4 to chemoradiotherapy (downstaging). 


\begin{tabular}{|c|c|c|c|c|c|c|}
\hline \multirow[t]{2}{*}{ Characteristics } & \multicolumn{4}{|c|}{ Downstaging } & \multirow[t]{2}{*}{ Total } & \multirow[t]{2}{*}{$p$-value ${ }^{(1)}$} \\
\hline & \multicolumn{2}{|c|}{\begin{tabular}{|l|} 
No \\
$\mathrm{N}=\mathbf{2 5}$
\end{tabular}} & \multicolumn{2}{|c|}{$\begin{array}{l}\text { Yes } \\
\mathrm{N}=96\end{array}$} & & \\
\hline & & & & & & \\
\hline Male & 16 & $22.9 \%$ & 54 & $77.1 \%$ & 70 & \\
\hline Female & 9 & $17.6 \%$ & 42 & $82.4 \%$ & 51 & \\
\hline Age (years) & & & & & & 0.12 \\
\hline Median - (Range) & 64.0 & $(28.0-80.0)$ & 61.5 & $(38.0-82.0)$ & $62.0 \quad(28.0-82.0)$ & \\
\hline Centre & & & & & & 0.99 \\
\hline Lille & 21 & $20.6 \%$ & 81 & $79.4 \%$ & 102 & \\
\hline Reims & 4 & $21.1 \%$ & 15 & $78.9 \%$ & 19 & \\
\hline Performance Status & & & & & & 0.99 \\
\hline WHO-O & 21 & $21.2 \%$ & 78 & $78.8 \%$ & 99 & \\
\hline WHO-1 & 4 & $18.2 \%$ & 18 & $81.8 \%$ & 22 & \\
\hline Tumor site & & & & & & $0.22 / 0.36^{(2)}$ \\
\hline Low rectum & 14 & $24.1 \%$ & 44 & $75.9 \%$ & 58 & \\
\hline Middle rectum & 8 & $14.5 \%$ & 47 & $85.5 \%$ & 55 & \\
\hline High rectum & 3 & $37.5 \%$ & 5 & $62.5 \%$ & 8 & \\
\hline Initial tumor size $(\mathrm{mm}), 1$ missing data & & & & & & 0.78 \\
\hline Median - (Range) & 45.0 & $(9.0-80.0)$ & 50.0 & $(4.5-120.0)$ & $50.0 \quad(4.5-120.0)$ & \\
\hline Tumor infiltration & & & & & & 0.85 \\
\hline Mesorectum & 21 & $21.0 \%$ & 79 & $79.0 \%$ & 100 & \\
\hline Mucosal & 0 & $0.0 \%$ & 1 & $100.0 \%$ & 1 & \\
\hline Muscular & 3 & $25.0 \%$ & 9 & $75.0 \%$ & 12 & \\
\hline Regional organ (prostate/uterus) & 0 & $0.0 \%$ & 4 & $100.0 \%$ & 4 & \\
\hline Serosal & 1 & $25.0 \%$ & 3 & $75.0 \%$ & 4 & \\
\hline Initial tumor staging $(T)$ & & & & & & $0.66 / 0.71^{(3)}$ \\
\hline $\mathrm{T} 2$ & 3 & $25.0 \%$ & 9 & $75.0 \%$ & 12 & \\
\hline T3 & 22 & $21.0 \%$ & 83 & $79.0 \%$ & 105 & \\
\hline $\mathrm{T} 4$ & 0 & $0.0 \%$ & 4 & $100.0 \%$ & 4 & \\
\hline Initial nodal staging (N) & & & & & & $0.054 / 0.076^{(4)}$ \\
\hline NO & 8 & $36.4 \%$ & 14 & $63.6 \%$ & 22 & \\
\hline N1 & 12 & $14.5 \%$ & 71 & $85.5 \%$ & 83 & \\
\hline N2 & 5 & $33.3 \%$ & 10 & $66.7 \%$ & 15 & \\
\hline $\mathrm{Nx}$ & 0 & $0.0 \%$ & 1 & $100.0 \%$ & 1 & \\
\hline Initial staging $\mathrm{TNM}^{(5)}$ & & & & & & 0.18 \\
\hline T2NOMO & 1 & $25.0 \%$ & 3 & $75.0 \%$ & 4 & \\
\hline T2N1M0 & 2 & $25.0 \%$ & 6 & $75.0 \%$ & 8 & \\
\hline T3NOMO & 7 & $38.9 \%$ & 11 & $61.1 \%$ & 18 & \\
\hline T3N1M0 & 10 & $13.9 \%$ & 62 & $86.1 \%$ & 72 & \\
\hline T3N2MO & 5 & $35.7 \%$ & 9 & $64.3 \%$ & 14 & \\
\hline T3NxMO & 0 & $0.0 \%$ & 1 & $100.0 \%$ & 1 & \\
\hline T4N1M0 & 0 & $0.0 \%$ & 3 & $100.0 \%$ & 3 & \\
\hline T4N2M0 & 0 & $0.0 \%$ & 1 & $100.0 \%$ & 1 & \\
\hline Initial staging UICC & & & & & & 0.087 \\
\hline Stage 2 & 8 & $33.3 \%$ & 16 & $66.7 \%$ & 24 & \\
\hline Stage 3 & 17 & $17.5 \%$ & 80 & $82.5 \%$ & 97 & \\
\hline
\end{tabular}




\begin{tabular}{|l|ll|ll|ll|l|}
$\begin{array}{l}\text { Radscore }^{(6)} \\
\text { Median - (Range) }\end{array}$ & 0.78 & $(-1.09-2.72)$ & 1.57 & $(-1.28-5.11)$ & 1.37 & $(-1.28-5.11)$ & $<0.001$ \\
\hline
\end{tabular}

2 1) p-value of the comparison tests between the two groups (downstaging yes versus no) using an appropriate 3 test (Chi-square or Fisher exact test for categorical variables, Student t-test for continuous variables)

4 2) p-value of the comparison test between low or middle versus high rectum tumor site

5 3) p-value of the test of the distribution of $\mathrm{T} 2$ versus $\mathrm{T} 3$ or $\mathrm{T} 4$

6 4) p-value of the test of the distribution of No versus $N+$

$7 \quad 5)$ Staging according to the TNM $7^{\text {th }}$ edition

8 6) Distribution of Radscore between the two groups is illustrated by a boxplot in Appendix-Figure 3 
2 Table 2: Association of chemoradiotherapy, surgery, and response to treatment with

3 downstaging

\begin{tabular}{|c|c|c|c|c|c|c|c|}
\hline \multirow[t]{2}{*}{ Characteristics } & \multicolumn{4}{|c|}{ Downstaging } & \multirow{2}{*}{\multicolumn{2}{|c|}{ Total }} & \multirow[t]{2}{*}{$p$-value ${ }^{(1)}$} \\
\hline & \multicolumn{2}{|c|}{$\begin{array}{l}\mathrm{No} \\
\mathrm{N}=25\end{array}$} & \multicolumn{2}{|c|}{$\begin{array}{l}\text { Yes } \\
\mathrm{N}=96\end{array}$} & & & \\
\hline 45 or $46 \mathrm{~Gy}$ & 3 & $42.9 \%$ & 4 & $57.1 \%$ & 7 & & \\
\hline $50 \mathrm{~Gy}$ & & $19.3 \%$ & 92 & $80.7 \%$ & 114 & & \\
\hline Type of chemotherapy regimen & & & & & & & 0.34 \\
\hline capecitabine or LV5FU2 & 25 & $21.7 \%$ & 90 & $78.3 \%$ & 115 & & \\
\hline capecitabine + metformine & 0 & $0.0 \%$ & 6 & $100.0 \%$ & 6 & & \\
\hline $\begin{array}{l}\text { Time interval between CRT and su } \\
\text { (weeks) }\end{array}$ & & & & & & & \\
\hline Median - (Range) & & $(4.0-15.0)$ & 8.0 & $(2.0-19.0)$ & 8.0 & $(2.0-19.0)$ & 0.68 \\
\hline$\leq 7$ weeks & 7 & $21.2 \%$ & 26 & $78.8 \%$ & 33 & & 0.93 \\
\hline$>7$ weeks & 18 & $20.5 \%$ & 70 & $79.5 \%$ & 88 & & \\
\hline Type of surgery & & & & & & & 0.076 \\
\hline Abdominoperineal resection & 8 & $36.4 \%$ & 14 & $63.6 \%$ & 22 & & \\
\hline Total mesorectal excision & 17 & $17.2 \%$ & 82 & $82.8 \%$ & 99 & & \\
\hline Surgical margin $(\mathrm{mm})$ & & & & & & & 0.033 \\
\hline RO & 21 & $18.4 \%$ & 93 & $81.6 \%$ & 114 & & \\
\hline R1 & 4 & $57.1 \%$ & 3 & $42.9 \%$ & 7 & & \\
\hline Post-operative tumor staging (pT) & & & & & & & $\mid<0.001$ \\
\hline рто & 0 & $0.0 \%$ & 26 & $100.0 \%$ & 26 & & \\
\hline pT1 & 0 & $0.0 \%$ & 14 & $100.0 \%$ & 14 & & \\
\hline pT2 & 2 & $5.7 \%$ & 33 & $94.3 \%$ & 35 & & \\
\hline pT3 & 23 & $51.1 \%$ & 22 & $48.9 \%$ & 45 & & \\
\hline pTis & 0 & $0.0 \%$ & 1 & $100.0 \%$ & 1 & & \\
\hline Post-operative nodal staging (pN) & & & & & & & $<0.001$ \\
\hline pNO & 3 & $3.5 \%$ & 82 & $96.5 \%$ & 85 & & \\
\hline pN1 & 12 & $46.2 \%$ & 14 & $53.8 \%$ & 26 & & \\
\hline $\mathrm{pN} 2$ & 10 & $100.0 \%$ & 0 & $0.0 \%$ & 10 & & \\
\hline Post-operative staging ypTNM & & & & & & & \\
\hline урTONOMO & 0 & $0.0 \%$ & 24 & $100.0 \%$ & 24 & & $0.004^{(2)}$ \\
\hline урTON1MO & 0 & $0.0 \%$ & 2 & $100.0 \%$ & 2 & & \\
\hline урT1NOMO & 0 & $0.0 \%$ & 12 & $100.0 \%$ & 12 & & \\
\hline урT1N1M0 & 0 & $0.0 \%$ & 2 & $100.0 \%$ & 2 & & \\
\hline урT2NOMO & 0 & $0.0 \%$ & 28 & $100.0 \%$ & 28 & & \\
\hline урT2N1M0 & 1 & $16.7 \%$ & 5 & $83.3 \%$ & 6 & & \\
\hline урT2N2M0 & 1 & $100.0 \%$ & 0 & $0.0 \%$ & 1 & & \\
\hline урT3NOMO & 3 & $15.0 \%$ & 17 & $85.0 \%$ & 20 & & \\
\hline урT3N1M0 & 11 & $68.8 \%$ & 5 & $31.3 \%$ & 16 & & \\
\hline урT3N2M0 & 9 & $100.0 \%$ & 0 & $0.0 \%$ & 9 & & \\
\hline ypTisNOMO & 0 & $0.0 \%$ & 1 & $100.0 \%$ & 1 & & \\
\hline
\end{tabular}

4 1) p-value of the comparison tests between the two groups (downstaging yes versus no)

5 2) p-value of the comparison test between ypTONOMO versus other 
1 Table 3: Univariable and multivariable analyses of downstaging in the training set (79

\begin{tabular}{|c|c|c|c|c|c|}
\hline \multirow{2}{*}{\multicolumn{2}{|c|}{ Characteristics }} & \multicolumn{2}{|c|}{ Univariate analysis } & \multicolumn{2}{|c|}{ Multivariate analysis* } \\
\hline & & OR (95\% Cl) & p-value & OR $(95 \% \mathrm{Cl})$ & p-value \\
\hline \multicolumn{2}{|c|}{ Radscore } & $7.96(2.30-31.98)$ & $<0.001$ & $13.25(4.06-71.64)$ & $<0.001$ \\
\hline \multicolumn{2}{|c|}{ Age (year) } & $1.05(1.00-1.10)$ & 0.054 & $1.10(1.03-1.20)$ & 0.008 \\
\hline \multicolumn{2}{|c|}{ Initial tumor size $(\mathrm{cm})$} & $1.19(0.95-1.55)$ & 0.140 & $1.00(0.71-1.43)$ & $0.99 * *$ \\
\hline $\begin{array}{c}\text { Initial tumor } \\
\text { staging }\end{array}$ & $\begin{array}{c}\text { T2 } \\
\text { T3-4 }\end{array}$ & $\begin{array}{c}1 \\
1.68(0.33-6.95) \\
\end{array}$ & 0.489 & & \\
\hline $\begin{array}{l}\text { Initial nodal } \\
\text { staging }\end{array}$ & $\begin{array}{l}\text { No } \\
\text { N+ }\end{array}$ & $\begin{array}{c}1 \\
2.45(0.66-8.54)\end{array}$ & 0.163 & $\begin{array}{c}1 \\
0.98(0.17-4.97)\end{array}$ & $0.98^{* *}$ \\
\hline $\begin{array}{c}\text { Initial } \\
\text { staging }\end{array}$ & $\begin{array}{l}\text { Stage } 2 \\
\text { Stage } 3\end{array}$ & $\begin{array}{c}1 \\
2.17(0.59-7.38)\end{array}$ & 0.223 & & \\
\hline $\begin{array}{c}\text { Time } \\
\text { interval } \\
\text { CRT-Surgery }\end{array}$ & $\begin{array}{l}\leq 7 \text { weeks } \\
>7 \text { weeks }\end{array}$ & $\begin{array}{c}1 \\
1.10(0.31-3.48)\end{array}$ & 0.871 & & \\
\hline Tumor site & $\begin{array}{c}\text { Low/Middle } \\
\text { High }\end{array}$ & $\begin{array}{c}1 \\
0.66(0.13-4.91)\end{array}$ & 0.636 & & \\
\hline $\begin{array}{c}\text { Performance } \\
\text { status }\end{array}$ & $\begin{array}{l}\text { WHO } 0 \\
\text { WHO } 1\end{array}$ & $\begin{array}{c}1 \\
2.19(0.53-14.94) \\
\end{array}$ & 0.335 & & \\
\hline
\end{tabular}

6

7 OR $(95 \% \mathrm{Cl})$ : odds ratio (95\% confidence interval)

$8 \quad$ * Backward step-wise selection was applied by using Akaike's information criterion as

9 the stopping rule. The final model includes only Radscore and age, leading to the 10 corresponding estimates (OR, 95\% Cl, and p-value).

$11^{* *}$ In the multivariable model, the estimates $(\mathrm{OR}, 95 \% \mathrm{Cl}$, and $\mathrm{p}$-value) for initial 12 tumor size and initial nodal staging were computed by separately adding each 13 variable to the final model.

14 RadScore, age, and initial tumor size were entered in the model as continuous 15 variables, after checking the loglinearity. The estimated odds ratios correspond to a 16 1-unit increment (OR associated with a 1 unit of Radscore, a 1 year of age, or with 1 $17 \mathrm{~cm}$ for tumor size). 
Figure 1

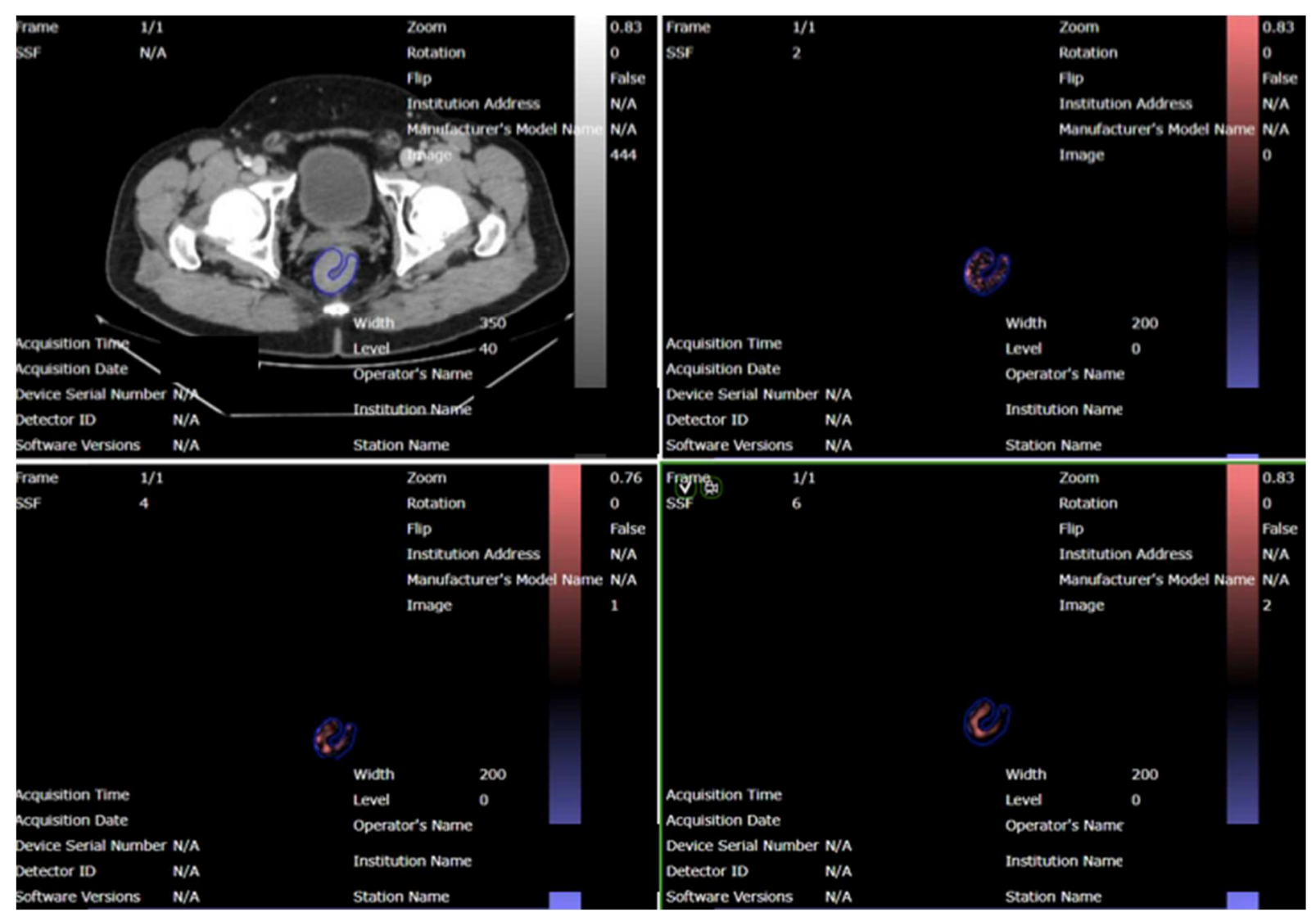


Figure 2

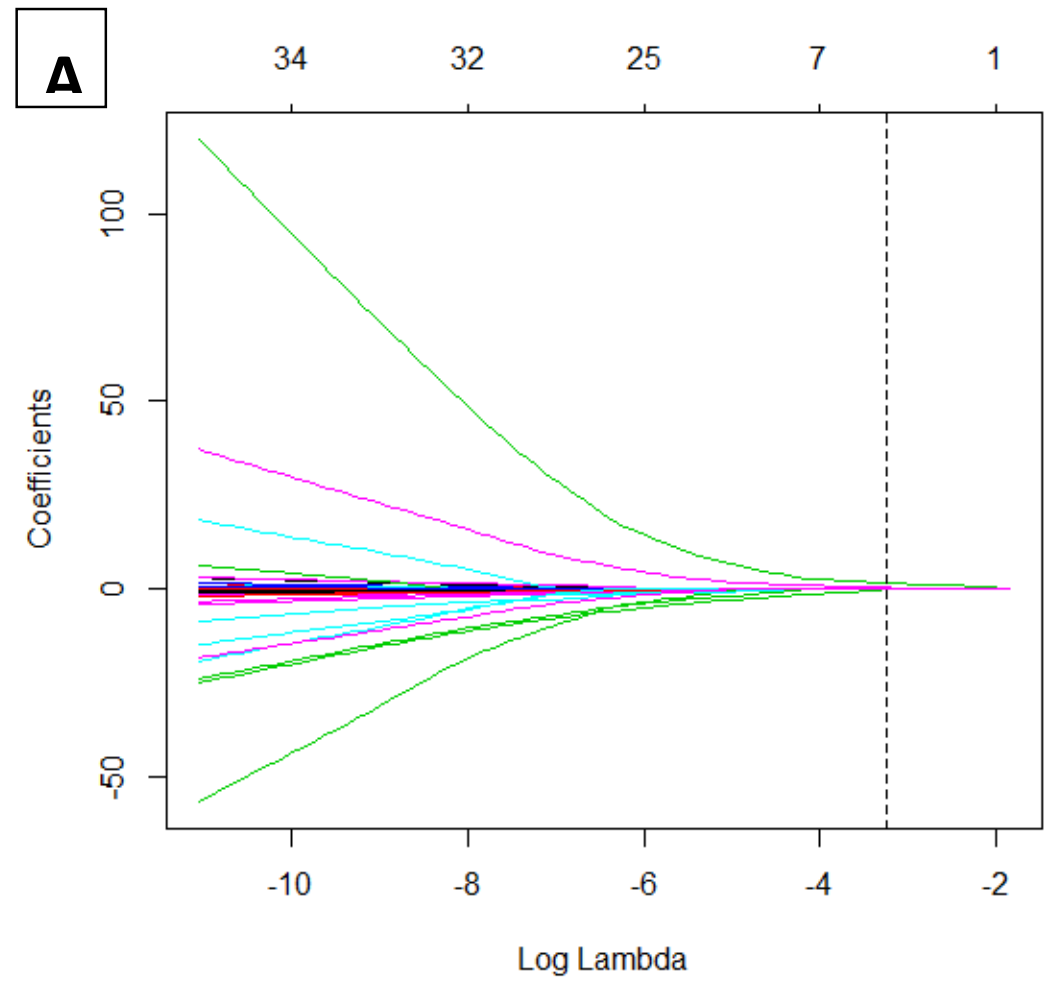

$\begin{array}{lllllllllllllll}\mathbf{R} & 34 & 34 & 33 & 32 & 31 & 30 & 27 & 24 & 14 & 7 & 7 & 5 & 2 & 0\end{array}$

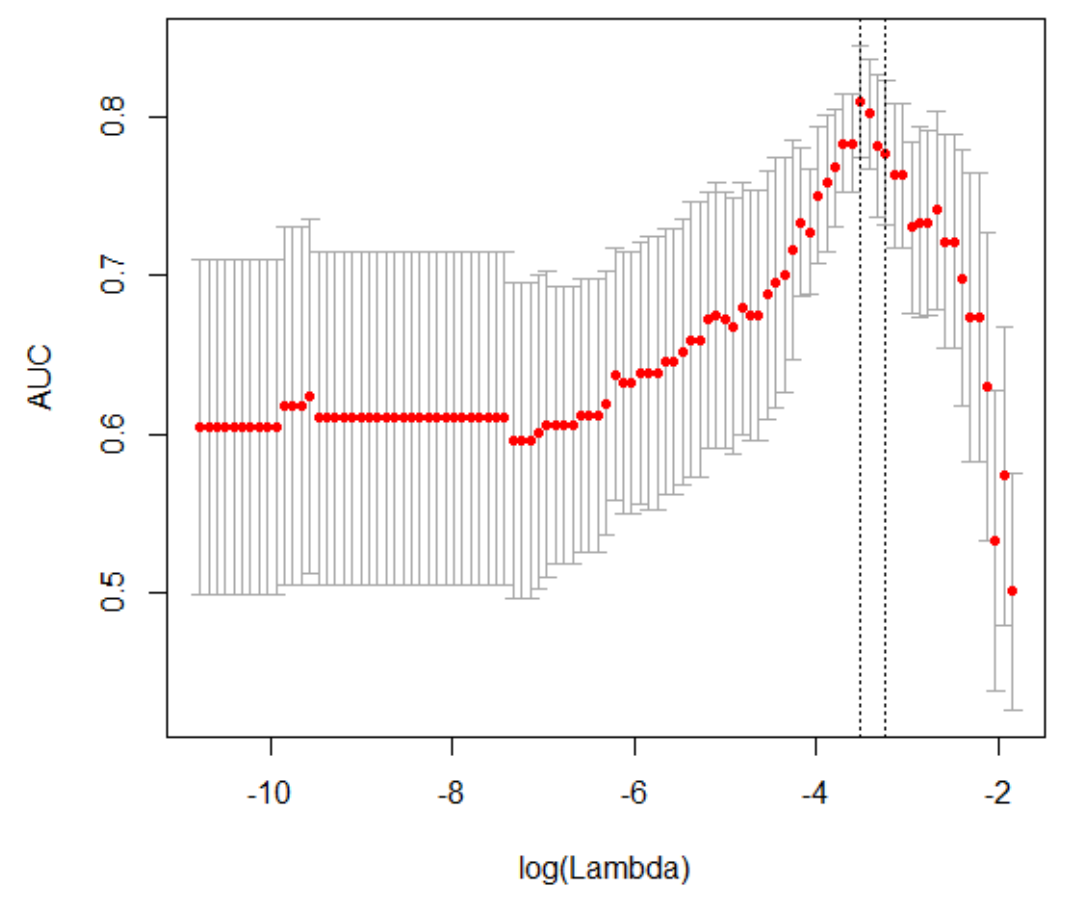


Figure 3

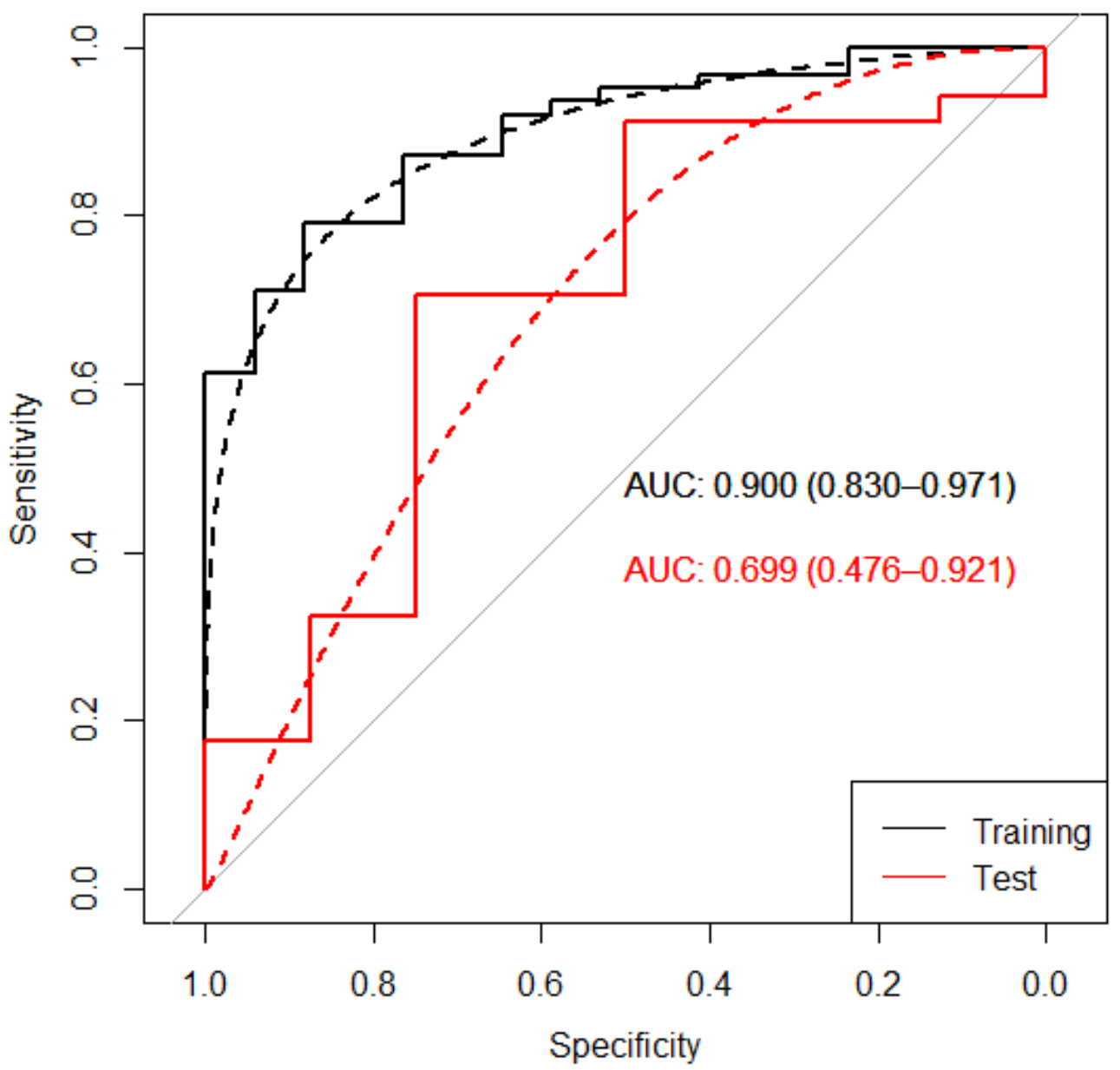

